\title{
Aplicações da modelagem de equações estruturais na enfermagem: revisão integrativa
}

Juliane Umann ${ }^{1}$, Rodrigo Marques da Silva ${ }^{2}$, Cristilene Akiko Kimura ${ }^{3}$, Liana Lautert ${ }^{4}$

\author{
${ }^{1}$ Enfermeira, Doutora em Enfermagem. \\ Enfermeira do Serviço Militar Brasileiro. São \\ Gabriel, RS, Brasil. E-mail: \\ juumann@hotmail.com. \\ ${ }^{2}$ Enfermeiro, Doutor em Enfermagem na \\ Saúde do Adulto. Professor da Faculdade de \\ Ciências e Educação Sena Aires. Valparaiso de \\ Goiás, GO, Brasil. E-mail: marques- \\ sm@hotmail.com. \\ ${ }^{3}$ Enfermeira, Doutora em Enfermagem. \\ Professora da Faculdade de Ciências e \\ Educação Sena Aires. Valparaiso de Goiás, GO, \\ Brasil. E-mail: cris.akiko7@gmail.com. \\ ${ }^{4}$ Enfermeira, Doutora em Psicologia. \\ Professora Associada da Universidade Federal \\ do Rio Grande do Sul. Porto Alegre, RS, Brasil. \\ E-mail: lila@enf.ufrgs.br.
}

Recebido: 29/06/2016.

Aceito: 22/09/2017.

Publicado: 31/12/2017.

Como citar esse artigo:

Umann J, Silva RM, Kimura CA, Lautert L. Aplicações da modelagem de equações estruturais na enfermagem: revisão integrativa. Rev. Eletr. Enf. [Internet]. 2017 [acesso em:____];19:a51. Disponível em: http://doi.org/10.5216/ree.v19.42988.

\begin{abstract}
RESUMO
Analisou-se as produções científicas que utilizaram a modelagem de equações estruturais em enfermagem. Trata-se de uma revisão integrativa realizada em junho de 2016 nas interfaces PUBMED, MEDLINE e LILACS. Identificaram-se 127 artigos, dos quais 20 artigos foram selecionados. As análises - qualidade e nível de evidência foram realizadas por meio de instrumentos validados e quadro sinóptico. Os artigos atenderam a 80\% dos itens do STROBE (95\%), nível de evidência 5 (95\%) e publicados em países da Ásia (50\%) e América do Norte (30\%). Houve aumento da produção cientifica com uso da modelagem de equações estruturais no período em estudo e o predomínio de investigações voltadas à organização do trabalho. Cresce o uso da modelagem de equações estruturais na enfermagem, contudo são escassos os estudos voltados à assistência e ensino. Este método mostrou-se útil para problemas de pesquisa desta área da saúde.
\end{abstract}

Descritores: Pesquisa em Enfermagem; Métodos; Análise de Dados; Análise Multivariada.

\section{INTRODUÇÃO}

O processo de trabalho em enfermagem envolve o Assistir, Administrar, Ensinar e Pesquisar, entretanto é a pesquisa que permite a melhora dos demais processos, à medida que fornece dados e instrumentos para qualificar a formação dos profissionais, a administração do cuidado e em consequência a assistência prestada. Destaca-se também que a pesquisa tem se fortalecido gradativamente nos últimos anos e contribuído para o corpus de conhecimento da enfermagem.

Na pesquisa da enfermagem observa-se o uso crescente de diferentes instrumentos e em especial as escalas psicométricas para mensuração de variáveis psicológicas. Concomitantemente também se verifica o 
emprego da análise estatística multivariada, permitindo testes empíricos robustos, uma vez que admite o exame de inúmeras relações simultaneamente, o que é coerente com a complexidade de alguns objetos de estudo. Entretanto, o manejo desses instrumentos e procedimentos exige cautela do pesquisador para realizar as análises e interpretações adequadas e evitar a falácia em pesquisa.

Um dos desenhos de pesquisa que tem se destacado na área da saúde são os modelos de equações estruturais (MEE). Estes permitem o teste das estruturas fatoriais de escalas psicométricas, por meio da análise fatorial confirmatória bem como análises de regressão. Assim o MEE permite confirmar a estrutura psicométrica de escalas para determinada amostra e também analisar relações explicativas entre múltiplas variáveis simultaneamente, sejam essas latentes ou observadas ${ }^{(1-2)}$.

A modelagem de equações estruturais, por se tratar de uma técnica confirmatória, envolve os quatro estágios do processo de Análise Fatorial Confirmatória voltada ao modelo de mensuração (1- Definição dos constructos individuais; 2- Desenvolvimento do modelo de mensuração geral; 3- Planejamento do estudo para produzir resultados empíricos; 4- Avaliação da validade do modelo de mensuração), bem como dois estágios específicos voltados ao modelo estrutural (5- Especificação do Modelo Estrutural; e 6- Avaliação da validade do modelo estrutural) ${ }^{(2)}$.

Uma etapa importante para uso da MEE é a definição do modelo a ser testado, ou seja, o estabelecimento a priori das relações entre as variáveis do modelo. A definição do modelo é norteada pela combinação de noções teóricas e evidências empíricas de investigações anteriores. Assim, para o emprego da MEE é imperioso considerar os aspectos teóricos e a elaboração/especificação de um modelo (relações hipotéticas entre as variáveis), bem como a representação gráfica do modelo hipotético e a fixação da escala de medida do modelo de mensuração ${ }^{(1-2)}$. E ainda, os dados em análise devem respeitar os cálculos de normalidade univariada e multivariada para a estimação ${ }^{(2)}$.

Deste modo, a MEE pode aprimorar os modelos de mensuração, auxiliar a elaboração de instrumentos mais adequados, o que beneficia o desenvolvimento de modelos teóricos eficientes para a descrição e explicação de fenômenos humanos e sociais.

Para a construção do modelo teórico a ser testado com a MEE, é preciso que o pesquisador certifiquese de que há associação suficiente entre duas variáveis; antecedência temporal da causa sobre o efeito; ausência de variáveis causais alternativas e referencial teórico que sustente as relações propostas ${ }^{(1)}$.

No entanto, o desenvolvimento de modelos teóricos explicativos, realizado por meio da MEE, ainda é recente e pouco usual na enfermagem ${ }^{(3-4)}$. Por isso, pouco se sabe sobre a aplicabilidade dessa técnica nos campos de ensino, assistência e administração, ou seja, como esse método pode contribuir para a construção e validação de modelos explicativos de inter-relações entre as variáveis de interesse do pesquisador.

Nesse sentido, esse estudo teve por objetivo analisar as produções científicas que utilizam a Modelagem de Equações Estruturais em enfermagem. 


\section{MÉTODOS}

Trata-se de uma revisão integrativa da literatura que busca evidências científicas por meio da revisão de múltiplos estudos publicados e permite a síntese para conclusões sobre o tema de estudo ${ }^{(5)}$. Para isso, realizaram-se as definições, do problema de pesquisa, da questão norteadora, dos descritores, dos critérios de inclusão e exclusão das publicações e das bases de dados. Na sequencia foram selecionados os artigos para extração, organização e sumarização das informações; avaliação dos estudos; interpretação dos resultados e síntese do conhecimento ${ }^{(5)}$. Com base nesse delineamento, elaborou-se a seguinte questão de pesquisa: Quais as aplicações de Modelagem de Equações Estruturais na pesquisa em enfermagem?

A captura de artigos científicos ocorreu em junho de 2016 na interface PUBMED e nas bases de dados MEDLINE e LILACS, utilizando-se a "Structural Equation Modeling"[Palavra do texto] AND "Nursing" [Descritor MESH] no formulário avançado dessas bases. Foram utilizados os seguintes filtros: publicações dos últimos cinco anos (2012-2015), tendo em vista a recente disseminação desta técnica na enfermagem no período e atualidade cientifica de publicações, texto disponível integralmente online e limite direcionado a humanos. Destaca-se que, embora a Medline seja uma base da interface Pubmed, as indexações dos artigos pelos periódicos não ocorrem simultaneamente nesses locais após a publicação nos periódicos. Por isso, a busca ocorreu no Pubmed e na base Medline (acesso via Biblioteca Virtual em Saúde) separadamente. Foram incluídos estudos originais, que usaram a Modelagem de Equações Estruturais na área de enfermagem e publicados em língua inglesa, espanhola e/ou portuguesa. Excluíram-se publicações que, mesmo com estratégia de captação prevista, estiveram fora do tema proposto, indisponíveis na íntegra e texto completo em outro idioma.

Após a captura, realizou-se a seleção inicial dos artigos por meio da leitura de títulos e resumos, tendo como critério inicial a coerência ao tema. Posteriormente estes foram analisados na íntegra segundo os critérios preestabelecidos. Para coleta e organização dos dados utilizou-se um instrumento validado em revisão integrativa sobre prevenção de lesões de pele nos pacientes em perioperatório ${ }^{(6)}$. Esse compõe-se pelos seguintes elementos: identificação do artigo original, características metodológicas, avaliação do rigor metodológico, das intervenções mensuradas e dos resultados encontrados. Para essa pesquisa, excluiu-se o item intervenção e foram utilizados itens adicionais, conforme segue - ano da publicação, objetivo, tamanho amostral, sujeitos de pesquisa, especialidade da enfermagem em que a MEE foi aplicada e nível de evidência de cada pesquisa. Os artigos selecionados foram identificados pela letra $A$ seguida de numeração arábica segundo a ordem em que foram citados.

A qualidade dos estudos da amostra final foi avaliada por meio da lista de verificação - versão traduzida para o português (Brasil): Strengthening the Reporting of Observational Studies in Epidemiology (STROBE). Optou-se pelo STROBE porque esse é considerado o atual padrão-ouro quando se trata de guiar a construção e avaliação de estudos observacionais. O STROBE é composto por 22 itens e objetiva auxiliar na condução e avaliação de estudos observacionais, incluindo estudos de coorte, caso-controle e estudos seccionais ${ }^{(7)}$. Do total de 22 itens, 18 são comuns a todos os delineamentos e quatro são específicos para cada desenho de 
estudo $^{(7)}$. A avaliação da qualidade das investigações foi realizada por dois revisores independentes de maneira cega, sendo mantidos aquelas que atenderam pelo menos $80 \%$ dos itens do STROBE.

Os estudos com mais de $80 \%$ dos itens propostos no STROBE foram inseridos em um banco de dados contendo as informações extraídas com base no instrumento validado e supracitado ${ }^{(6)}$. Para a análise das evidências dos estudos, foi utilizada a classificação proposta por Melnyk e Fineout-Overholt ${ }^{(8)}$. Nesta, a qualidade das evidências é classificada em seis níveis, a saber:

- Nível 1: evidências oriundas de revisões sistemáticas ou metanálise de relevantes ensaios clínicos;

- Nível 2: evidências derivadas de pelo menos um ensaio clínico randomizado controlado bem delineado;

- Nível 3: ensaios clínicos bem delineados sem randomização;

- Nível 4: estudos de coorte e de caso-controle bem delineados;

- Nível 5: revisão sistemática de estudos descritivos e qualitativos;

- Nível 6: evidências derivadas de um único estudo descritivo ou qualitativo;

- Nível 7: opinião de autoridades ou relatório de comitês de especialistas.

\section{RESULTADOS}

Identificaram-se 127 artigos com a estratégia de busca proposta. Após a leitura dos títulos e resumos (pré-seleção), foram excluídos 100 artigos que usaram a MEE sem ênfase na enfermagem. Na leitura dos artigos na íntegra, dois artigos do Pubmed estavam em Coreano e cinco do Medline indisponíveis na íntegra, o que levou a uma composição final de 20 artigos (Figura 1). 


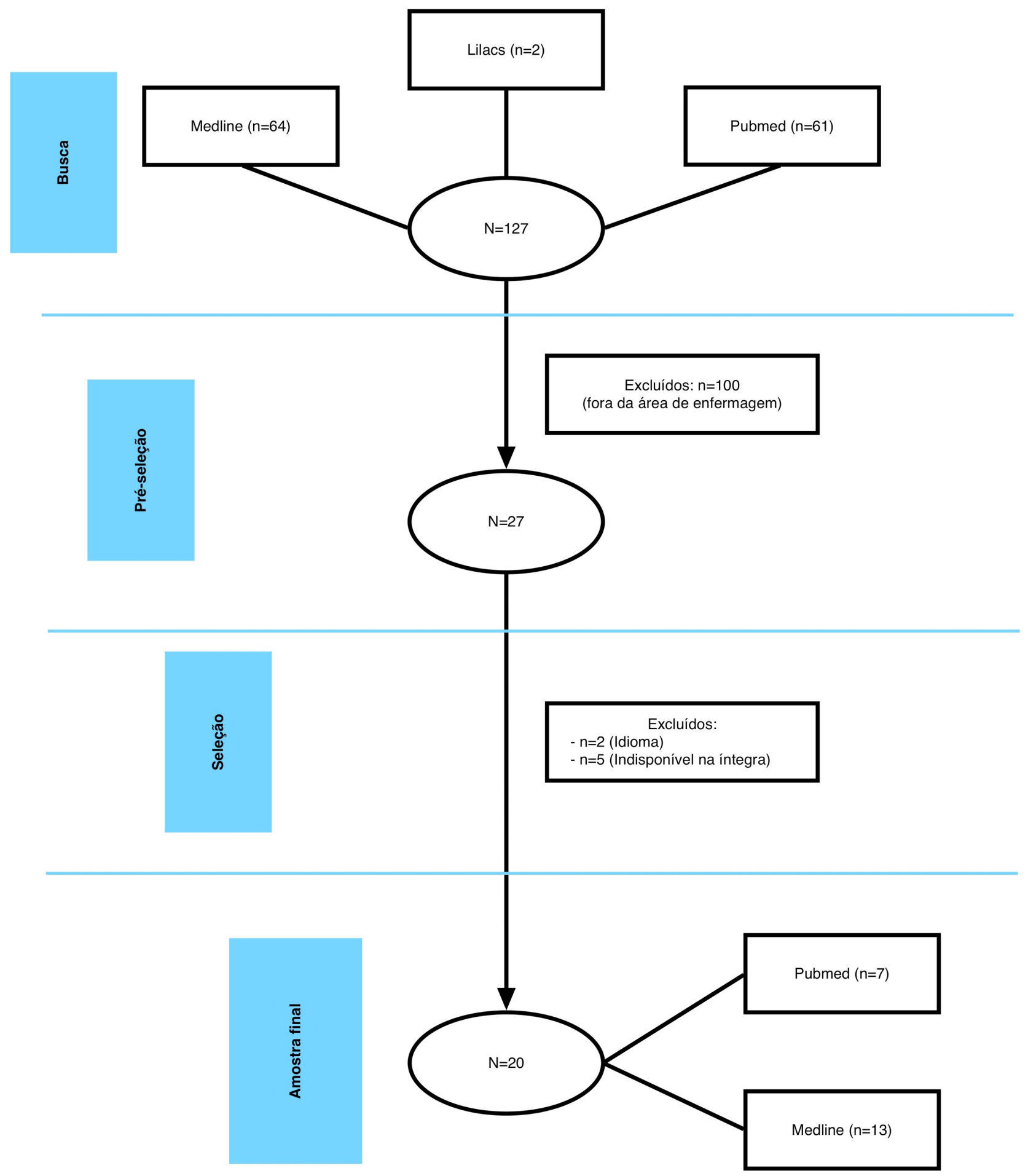

Figura 1: Fluxo de exclusão dos estudos selecionados na revisão.

Avaliação de qualidade e caracterização da produção científica

Na análise da qualidade da produção, 19 artigos (95\%) contemplaram mais de $80 \%$ dos itens propostos no STROBE. Um artigo atendeu $70 \%$ dos itens, mas se optou por mantê-lo na revisão porque os itens adequados pertenciam a seções fundamentais do estudo, a ser: objetivo, hipótese e método empregado (Quadro 1). 
Quadro 1: Quadro sinóptico das produções científicas incluídas na revisão, 2016.

\begin{tabular}{|c|c|c|c|c|}
\hline Ref & Objetivo & Resultados & Amostra / País & $\begin{array}{l}\text { STROBE* } \\
\text { Evidência }\end{array}$ \\
\hline $\mathrm{A} 1^{(9)}$ & $\begin{array}{l}\text { Investigar como a percepção de justiça organizacional afeta a } \\
\text { confiança e identificação organizacional de enfermeiras, e se a } \\
\text { confiança e identificação organizacional encorajam as } \\
\text { enfermeiras a voluntariamente permanecer no trabalho e a } \\
\text { comprometer-se com os hospitais. }\end{array}$ & $\begin{array}{l}\text { A justiça organizacional percebida pelas enfermeiras influencia positivamente } \\
\text { sua confiança organizacional }\left(\gamma_{11}=0.49\right) \text { e a identificação organizacional } \\
\left(\gamma_{21}=0.58\right) \text {. A confiança }\left(\beta_{31}=0.62\right) \text { e a identificação organizacional afetam } \\
\text { positivamente o compromisso com a instituição }\left(\beta_{32}=0.53\right) \text {. }\end{array}$ & $\begin{array}{l}386 \text { Enfermeiras / } \\
\text { Estados Unidos da } \\
\text { América }\end{array}$ & 18 \\
\hline$A 2^{(10)}$ & $\begin{array}{l}\text { Avaliar a eficácia das estratégias, clássicas (disponibilidade de } \\
\text { produtos a base de álcool, pôster, instruções e formação) ou } \\
\text { avançadas (feedbacks e liderança formal e informal), para } \\
\text { melhorar o cumprimento da higienização das mãos. }\end{array}$ & $\begin{array}{l}\text { A confiabilidade dos constructos propostos foi obtida (Cronbach } \alpha=0,73 ; 0,84 ; \\
0,70 \text { ) e verificou-se que os profissionais de saúde que atuam em centros mais } \\
\text { comprometidos com a higienização das mãos são aqueles que percebem as } \\
\text { estratégias avançadas como mais efetivas ( } \chi 2=298,3, \mathrm{df}=39, \mathrm{CFI}=0,972, \\
\mathrm{TLI}=0,961, \mathrm{RMSEA}=0,057, \mathrm{SRMR}=0,028) .\end{array}$ & $\begin{array}{l}\text { 2.068 Profissionais de } \\
\text { saúde / Espanha }\end{array}$ & 19 \\
\hline$A 3^{(11)}$ & $\begin{array}{l}\text { Testar um modelo para predizer e explicar a efetividade no } \\
\text { trabalho de enfermeiras dos hospitais locais. }\end{array}$ & $\begin{array}{l}\text { O modelo de caminhos final mostrou um bom ajuste dos dados, gerando um } \\
\text { modelo modificado. As características do trabalho e a possibilidade de } \\
\text { indenização judicial (por danos) apresentaram efeito positivo sobre o } \\
\text { empoderamento. As características do trabalho, o empoderamento e a } \\
\text { liderança transformacional afetam, direta e positivamente, a efetividade no } \\
\text { trabalho. As características laborais apresentaram um efeito maior sobre o } \\
\text { empoderamento e efetividade no trabalho do que os demais fatores } \\
\text { estudados. }\end{array}$ & $\begin{array}{l}340 \text { Enfermeiras / } \\
\text { Coréia do Sul }\end{array}$ & 18 \\
\hline $\mathrm{A} 4^{(12)}$ & $\begin{array}{l}\text { Avaliar um modelo baseado na teoria da autoeficácia para } \\
\text { compreensão do desempenho profissional de enfermeiras } \\
\text { relativo ao controle de peso de pacientes obesos. }\end{array}$ & $\begin{array}{l}\text { A autoeficácia direta e positivamente predisse as práticas de controle de peso } \\
\text { das enfermeiras }(\beta=0.36, p<0.01) \text {, e mediou as relações entre habilidades } \\
\text { percebidas, barreiras percebidas, identidade profissional, crença no trabalho } \\
\text { em equipe e práticas de controle de peso. O modelo final demonstrou um bom } \\
\text { ajuste aos dados }[\chi 2(14)=13,90, p=0,46 ; \mathrm{GFI}=0,99 ; \mathrm{AGFI}=0,98 ; \mathrm{NNFI}=1,00 ; \\
\mathrm{CFI}=1,00 ; \mathrm{RMSEA}=0,00 ; \mathrm{AIC}=57,90] \text {, contribuindo para } 38.4 \% \text { e } 43.2 \% \text { da } \\
\text { variância nos fatores práticas de controle de peso e autoeficácia } \\
\text { respectivamente. }\end{array}$ & $\begin{array}{l}399 \text { Enfermeiras / } \\
\text { Reino Unido }\end{array}$ & 17 \\
\hline$A 5^{(13)}$ & $\begin{array}{l}\text { Investigar efeito de preparação tecnológica dos enfermeiros } \\
\text { sobre a aceitação de sistemas eletrônicos móveis de registro } \\
\text { médico (SEMRM) }\end{array}$ & $\begin{array}{l}\text { Os resultados apontam que as enfermeiras são otimistas, inovadoras e seguras, } \\
\text { mas se sentem desconfortáveis em relação à tecnologia. Além disso, esses } \\
\text { quatro traços de preparação tecnológica apresentaram impacto significativo } \\
\text { sobre a facilidade de uso percebida dos SEMRM, enquanto a utilidade } \\
\text { percebida dos SEMRM foi significantemente influenciada pelo traço de } \\
\text { otimismo. Os resultados também confirmaram as relações entre a facilidade de } \\
\text { uso, a utilidade e a intenção comportamental no Modelo de Aceitação } \\
\text { Tecnológica frente ao uso do SEMRM. }\end{array}$ & $\begin{array}{l}665 \text { Enfermeiras } \\
\text { hospitalares / Taiwan }\end{array}$ & 17 \\
\hline
\end{tabular}

Rev. Eletr. Enf. [Internet]. 2017 [acesso em:_____];19:a51. Disponível em: http://doi.org/10.5216/ree.v19.42988. 


\begin{tabular}{|c|c|c|c|c|}
\hline Ref & Objetivo & Resultados & Amostra / País & $\begin{array}{l}\text { STROBE* } \\
\text { Evidência }\end{array}$ \\
\hline$A 6^{(14)}$ & $\begin{array}{l}\text { Avaliar as características do ambiente da prática profissional } \\
\text { dos enfermeiros e sua relação com o Burnout, percepção da } \\
\text { qualidade do cuidado, satisfação no trabalho e a intenção de } \\
\text { deixar o emprego nos próximos doze meses. }\end{array}$ & $\begin{array}{l}\text { A modelagem de equações estruturais, por meio da análise de caminhos, } \\
\text { mostrou que as características do ambiente da prática influenciam a satisfação } \\
\text { no trabalho, percepção da qualidade do cuidado e a intenção de deixar o } \\
\text { emprego, quando mediadas pelo sentimento de exaustão emocional. o } \\
\text { enfermeiro com pouca autonomia, menor controle sobre o ambiente e com } \\
\text { piores relações com o médico, apresenta maior exaustão emocional, o que } \\
\text { pode influenciar negativamente na sua percepção da qualidade do cuidado, } \\
\text { satisfação com o trabalho e na intenção de deixar o emprego. }\end{array}$ & $\begin{array}{l}129 \text { Enfermeiras de } \\
\text { UTI adulto / Brasil }\end{array}$ & 18 \\
\hline$A 7^{(15)}$ & $\begin{array}{l}\text { Investigar se a capacidade percebida de aprendizagem } \\
\text { organizacional (CAO) associa-se à aceitação da tecnologia de } \\
\text { informação entre a equipe de enfermagem cirúrgica. }\end{array}$ & $\begin{array}{l}\text { A expectativa de desempenho e de esforço e a influência social contribuíram } \\
\text { positivamente ( } p<0.001 \text { ) para intenção comportamental das usuárias do } \\
\text { sistema de informação clínica, explicando } 75 \% \text { da variância. A CAO se associou } \\
\text { positivamente a expectativa de desempenho, de esforço e a influência social } \\
(p<0.001) \text {. Todavia, a relação hipotética entre CAO percebida e intenção } \\
\text { comportamental não foi significativa }(p=0.87) \text {. A análise estatística de ajuste } \\
\text { indicou um ajuste razoável do modelo aos dados (Raiz do erro quadrático } \\
\text { médio de aproximação }=0,07 \text { e Índice de Ajuste Comparativo= } 0,91 \text { ). }\end{array}$ & $\begin{array}{l}84 \text { Enfermeiras } \\
\text { Anestesistas e } 131 \\
\text { Enfermeiras de Centro } \\
\text { Cirúrgico / Taiwan }\end{array}$ & 17 \\
\hline$A 8^{(16)}$ & $\begin{array}{l}\text { Avaliar como a identificação das enfermeiras com seu grupo de } \\
\text { trabalho, unidade ou andar; a função exercida; e a carreira de } \\
\text { enfermagem se relacionam à capacidade de interação, à } \\
\text { disposição para manejar conflitos, ao sentimento de } \\
\text { desamparo e à estabilidade laboral. }\end{array}$ & $\begin{array}{l}\text { Os achados demostram relações diretas entre a identificação da enfermeira } \\
\text { com a equipe (variável latente) e sua capacidade de interação, disposição em } \\
\text { manejar conflitos e estabilidade laboral. Sentimentos de desamparo são } \\
\text { atenuados pela maior identificação da enfermeira com a equipe por meio da } \\
\text { capacidade de interação e da disposição em manejar conflitos. Além disso, a } \\
\text { disposição em manejar conflitos e o desamparo mediam as relações entre } \\
\text { capacidade de interação e estabilidade laboral. }\end{array}$ & $\begin{array}{l}466 \text { Enfermeiras de } \\
\text { um hospital / Estados } \\
\text { Unidos da América }\end{array}$ & 17 \\
\hline$A 9^{(17)}$ & $\begin{array}{l}\text { Relacionar o estilo de liderança percebida(particularmente a } \\
\text { liderança percebida pelo funcionário) suporte organizacional } \\
\text { percebido, confiança no líder e na organização e Burnout entre } \\
\text { enfermeiras com a sua intenção de deixar o trabalho. }\end{array}$ & $\begin{array}{l}\text { A liderança percebida pelo funcionário foi positivamente associada a confiança } \\
\text { no líder; e o suporte organizacional percebido foi positivamente associado a } \\
\text { confiança na organização. A confiança no líder e na organização mostraram } \\
\text { correlação negativa com a exaustão emocional e cinismo, e positiva com a } \\
\text { eficácia profissional. Os escores de confiança mediaram os efeitos da liderança } \\
\text { do funcionário e suporte organizacional percebido sobre os fatores de Burnout. } \\
\text { O fator cinismo correlacionou-se negativamente à intenção de deixar o } \\
\text { trabalho; e mediou os efeitos da confiança no líder e da liderança percebida do } \\
\text { funcionário sobre a intenção de deixar o emprego. A confiança na organização } \\
\text { foi direta e negativamente relacionada à intenção de deixar o emprego. }\end{array}$ & $\begin{array}{l}711 \text { Membros da } \\
\text { equipe de } \\
\text { enfermagem de um } \\
\text { hospital de grande } \\
\text { porte / Itália }\end{array}$ & 20 \\
\hline
\end{tabular}




\begin{tabular}{|c|c|c|c|c|}
\hline Ref & Objetivo & Resultados & Amostra / País & $\begin{array}{l}\text { STROBE* } \\
\text { Evidência }\end{array}$ \\
\hline $\mathrm{A} 10^{(18)}$ & $\begin{array}{l}\text { Avaliar a satisfação com a efetividade do ambiente de } \\
\text { aprendizado clínico - AAC entre estudantes de enfermagem de } \\
\text { Oman. }\end{array}$ & $\begin{array}{l}\text { A satisfação apresentou correlação com as subdimensões do ambiente de } \\
\text { aprendizado clínico (AAC) e demonstrou relação positiva com o escore geral de } \\
\text { AAC. No modelo de caminhos, } 35 \% \text { da variância total da satisfação com a AAC } \\
\text { foi explicada pelo estilo de liderança, compromisso com a enfermagem clínica e } \\
\text { relacionamento com o paciente. A maior idade, o desempenho acadêmico } \\
\text { (CFA) e o número de disciplinas clínicas cursadas foram significativos para a } \\
\text { satisfação com a AAC entre esses estudantes. }\end{array}$ & $\begin{array}{l}310 \text { Estudantes de } \\
\text { enfermagem / Oman }\end{array}$ & 18 \\
\hline $\mathrm{A} 11^{(19)}$ & $\begin{array}{l}\text { Investigar as relações entre vários fatores do contexto } \\
\text { academico em uma amostra nacionalmente representativa de } \\
\text { docentes de enfermagem dos EUA. }\end{array}$ & $\begin{array}{l}\text { Embora diferentes efeitos (diretos e indireto) entre os fatores analisados } \\
\text { tenham sido observados, destaca-se que as percepções de suporte da } \\
\text { administração e a expertise percebida no ensino predizem positivamente a } \\
\text { intenção de professores de enfermagem de permanecerem nas instituições de } \\
\text { ensino investigadas. }\end{array}$ & $\begin{array}{l}657 \text { Docentes de } \\
\text { enfermagem / Estados } \\
\text { Unidos da América }\end{array}$ & 19 \\
\hline $\mathrm{A} 12^{(20)}$ & $\begin{array}{l}\text { Testar os determinantes e os efeitos da autorreflexão e do } \\
\text { insight sobre competência de estudantes de enfermagem } \\
\text { durante os dois primeiros meses da sua experiência prática. }\end{array}$ & $\begin{array}{l}\text { Os resultados indicaram que a autorreflexão e o insight, o estresse e o } \\
\text { enfrentamento das atividades práticas foram significativamente associados à } \\
\text { competência dos alunos de enfermagem. A autorreflexão e o insight } \\
\text { correlacionaram-se positivamente ao enfrentamento das atividades práticas e } \\
\text { negativamente ao estresse. Os comportamentos de enfrentamento mediaram } \\
\text { parcialmente os efeitos da autorreflexão e do estresse sobre a competência } \\
\text { clínica. No total, essas variáveis explicaram } 39,4 \% \text { da variância na competência } \\
\text { clínica dos estudantes. }\end{array}$ & $\begin{array}{l}312 \text { Acadêmicos de } \\
\text { enfermagem / Taiwan }\end{array}$ & 18 \\
\hline $\mathrm{A} 13^{(21)}$ & $\begin{array}{l}\text { Explicar a aceitação e o uso real de um sistema eletrônico de } \\
\text { registo de pacientes (ERP) e a satisfação de enfermeiras } \\
\text { testando um modelo teórico adaptado da Teoria Unificada de } \\
\text { Aceitação e Uso da Tecnologia (UTAUT) }\end{array}$ & $\begin{array}{l}13 \text { das } 20 \text { hipóteses propostas foram confirmadas. Os efeitos mais fortes foram } \\
\text { aqueles entre o desempenho esperado (produtividade) e o uso real do ERP ( } r= \\
0 ., 55, p=0,006) \text {, a facilitação das condições e o esforço esperado (para } \\
\text { aprender a usar o novo sistema) ( } r=0,45, p=0,009 \text { ), compatibilidade e } \\
\text { desempenho esperado }(r=0.39, p=0,002) \text {. As variáveis explicaram } 33,6 \% \text { da } \\
\text { variância do uso real, } 54,9 \% \text { da satisfação das enfermeiras, } 50,2 \% \text { do } \\
\text { desempenho esperado e } 52,9 \% \text { do esforço esperado }\end{array}$ & $\begin{array}{c}616 \text { Enfermeiras / } \\
\text { Canadá }\end{array}$ & 20 \\
\hline $\mathrm{A} 14^{(22)}$ & $\begin{array}{l}\text { Testar um modelo multinível avaliando o efeito dos fatores } \\
\text { individuais e contextuais sobre a satisfação do enfermeiro com } \\
\text { o trabalho. }\end{array}$ & $\begin{array}{l}\text { A percepção de empoderamento estrutural das enfermeiras por seus grupos } \\
\text { influenciou indiretamente a percepção de efetividade coletiva (Nível 2) por } \\
\text { meio do suporte coletivo percebido no exercício profissional, o que, por sua } \\
\text { vez, teve um efeito direto e positivo na efetividade coletiva (Nível 2). A } \\
\text { efetividade coletiva também se correlacionou à satisfação do enfermeiro com o } \\
\text { trabalho após um ano. No nível 1, escores mais altos de autoavaliação } \\
\text { produziram efeitos diretos e indiretos sobre a satisfação no 4trabalho por meio } \\
\text { do aumento do empoderamento psicológico. }\end{array}$ & $\begin{array}{c}545 \text { Enfermeiras / } \\
\text { Canadá }\end{array}$ & 19 \\
\hline
\end{tabular}

Rev. Eletr. Enf. [Internet]. 2017 [acesso em:_____];19:a51. Disponível em: http://doi.org/10.5216/ree.v19.42988. 


\begin{tabular}{|c|c|c|c|c|}
\hline Ref & Objetivo & Resultados & Amostra / País & $\begin{array}{l}\text { STROBE* } \\
\text { Evidência }\end{array}$ \\
\hline $\mathrm{A} 15^{(23)}$ & $\begin{array}{l}\text { Investigar as associações entre as habilidades de resolução de } \\
\text { problemas e o hardiness com o estresse percebido em } \\
\text { enfermeiras }\end{array}$ & $\begin{array}{c}\text { Altos escores de hardiness foram associados a baixos níveis de estresse } \\
\text { percebido. Enfermeiras com baixo estresse percebido apresentaram maior } \\
\text { chance de serem acessíveis; um estilo de controle pessoal interno; e confiança } \\
\text { efetiva para resolução de problemas. }\end{array}$ & 252 Enfermeiras / Irã & 17 \\
\hline $\mathrm{A} 16^{(24)}$ & $\begin{array}{l}\text { Explorar as relações entre o fator social, a autoeficácia com a } \\
\text { internet e as atitudes frente à formação continuada online no } \\
\text { contexto clínico de enfermagem. }\end{array}$ & $\begin{array}{l}\text { O fator social está relacionado à autoeficácia com a internet e as atitudes } \\
\text { frente à formação continuada online (incluindo utilidade percebida, facilidade } \\
\text { de uso percebida, e afeição). A autoeficácia com a internet básica apresenta um } \\
\text { papel fundamental sobre as atitudes, incluindo utilidade percebida, facilidade } \\
\text { de uso percebida, e afeição. No entanto, a autoeficácia com a internet } \\
\text { avançada não se correlacionou a nenhuma das atitudes. A dimensão } \\
\text { comportamento não se relacionou ao fator social e à autoeficácia com a } \\
\text { internet, mas se correlacionou com a facilidade de uso percebida e afeição. }\end{array}$ & $\begin{array}{l}244 \text { Enfermeiras / } \\
\text { Taiwan }\end{array}$ & 15 \\
\hline $\mathrm{A} 17^{(25)}$ & $\begin{array}{l}\text { Investigar os efeitos do estresse, fatores econômicos, altruísmo } \\
\text { e da congruência de valores nas intenções de abandoar o } \\
\text { trabalho e a profissão de enfermagem. }\end{array}$ & $\begin{array}{l}\text { Os resultados confirmam o efeito do estresse e da satisfação no trabalho sobre } \\
\text { a intenção de deixar a profissão de enfermagem, bem como destacam o } \\
\text { impacto da congruência de valores e do altruísmo sobre a satisfação no } \\
\text { trabalho. Os resultados ressaltam a importância da congruência de valores e } \\
\text { altruísmo em um campo em que o estresse e os fatores econômicos têm sido } \\
\text { considerados os fatores mais relevantes. }\end{array}$ & $\begin{array}{l}861 \text { Enfermeiras / } \\
\text { Estados Unidos da } \\
\text { América }\end{array}$ & 17 \\
\hline $\mathrm{A} 18^{(26)}$ & $\begin{array}{l}\text { Avaliar o papel das experiências dolorosas em relação à } \\
\text { ausência no trabalho, junto a outros fatores ocupacionais e de } \\
\text { saúde, em enfermeiras canadenses com distúrbios } \\
\text { musculoesqueléticos relacionados ao trabalho. }\end{array}$ & $\begin{array}{l}\text { O modelo final sugere que a severidade da dor e interferência da dor na } \\
\text { atividade laboral mediam o impacto dos seguintes fatores ocupacionais e de } \\
\text { saúde na duração da ausência no trabalho: depressão, distúrbios na coluna, } \\
\text { idade, sindicalização, demandas físicas no local de trabalho e baixo controle no } \\
\text { trabalho. O modelo explicou } 14 \% \text { da variância na duração da ausência no } \\
\text { trabalho e } 46,6 \% \text { da variância na interferência da dor no trabalho. }\end{array}$ & $\begin{array}{l}941 \text { Enfermeiras / } \\
\text { Canadá }\end{array}$ & 20 \\
\hline $\mathrm{A} 19^{(27)}$ & $\begin{array}{l}\text { Conhecer os fatores que levam aos erros de medicação } \\
\text { cometidos por estudantes de enfermagem. }\end{array}$ & $\begin{array}{l}\text { Cinco fatores causais de erros de medicação entre estudantes de enfermagem } \\
\text { foram identificados: violação (descumprimento dos protocolos), escrita, } \\
\text { excesso de demandas, experiência e tensão. Os resultados da análise } \\
\text { mostraram interação entre essas variáveis. A baixa aderência aos cinco certos } \\
\text { foi verificada como um importante mediador entre violação, escrita, excesso, } \\
\text { experiência e tensão e os erros de medicação cometidos pelos estudantes. }\end{array}$ & $\begin{array}{l}329 \text { Estudantes de } \\
\text { enfermagem / } \\
\text { Filipinas }\end{array}$ & 18 \\
\hline$A 20^{(28)}$ & $\begin{array}{l}\text { Investigar os fatores que afetam o ajuste entre as atividades de } \\
\text { enfermagem e o sistema móvel de informação em } \\
\text { enfermagem, e as relações entre a adequação tecnologia- } \\
\text { tarefa do sistema móvel e o desempenho de enfermeiras. }\end{array}$ & $\begin{array}{l}\text { Identificou-se que as funcionalidades de suporte oferecidas pelo sistema } \\
\text { desempenham efeito positivo na aquisição, interpretação e integração das } \\
\text { informações. Essas três variáveis também são positivamente afetadas pelo } \\
\text { serviço de suporte do sistema e contribuem para o desempenho das } \\
\text { enfermeiras, com } 83,2 \% \text { da variância total explicada. }\end{array}$ & $\begin{array}{l}219 \text { Enfermeiras / } \\
\text { Taiwan }\end{array}$ & 17 \\
\hline
\end{tabular}

Rev. Eletr. Enf. [Internet]. 2017 [acesso em:_____];19:a51. Disponível em: http://doi.org/10.5216/ree.v19.42988. 
Verificou-se predomínio de estudos (95\%) com Nível de Evidência 5, publicados em 2015 (30\%), em países da Ásia (50\%), América do Norte (30\%), Europa (15\%) e voltados à área da administração, mais precisamente ao processo de organização do trabalho de enfermagem (70\%).

Na classificação e análise dos temas de enfermagem abordados nas pesquisas que utilizaram a MEE, segundo o ano, observou-se que as publicações voltadas à assistência de enfermagem (dois), foram divulgadas em 2013 e 2014 enquanto que os artigos sobre o ensino na enfermagem (quatro) foram publicados em 2013 (um) e 2015 (três). Na área da administração de enfermagem/organização do trabalho, por sua vez, houve publicações durante todo período em estudo, ou seja, um, quatro, cinco e quatro artigos respectivamente entre 2012 e 2015. Por ano, o somatório das publicações foi de um, seis, seis e sete artigos no período (2012-2015).

No Quadro 1, apresenta-se o quadro sinóptico dessa revisão, incluindo objetivo, resultados, amostra/país e pontuação dos estudos selecionados segundo o STROBE.

\section{Aplicações do MEE na Enfermagem}

As publicações contemplam diferentes subáreas de conhecimento, que serão abordadas conforme foco: assistência de enfermagem, ensino e administração em enfermagem.

No que concerne à assistência de enfermagem ${ }^{(10,12)}$, os estudos em análise remetem a parâmetros de qualidade, sendo avaliado o efeito - do comprometimento organizacional sobre a efetividade de técnicas de higienização de mãos; e da teoria da autoeficácia no controle do peso de pacientes adultos. Os resultados dos estudos demonstram que o grau de comprometimento da organização, feedback e a liderança são fatores que contribuem para a adesão às práticas ampliadas de lavagem de mãos ${ }^{(10)}$ e a percepção de autoeficácia da enfermeira no trabalho estimula o controle do peso de pacientes obesos ${ }^{(12)}$.

Quanto ao ensino da enfermagem ${ }^{(18-20,27)}$ foram analisados entre estudantes os elementos preditores da efetividade de aprendizado, quais sejam: idade, desempenho acadêmico, número de disciplinas cursadas, liderança, compromisso com a enfermagem clínica e relacionamento com o paciente ${ }^{(18)}$; autoreflexão e insight ${ }^{(20)}$; enquanto erros de medicação foram atribuídos a violação dos protocolos, dificuldades com os registros clínicos, experiência, tensão e excesso de demandas no ambiente clínico ${ }^{(27)}$. Esses fatores produziram efeitos significativos sobre a efetividade e satisfação com a prática ${ }^{(18)}$, a competência percebida ${ }^{(20)}$ e os erros $^{(27)}$, respetivamente. Sobre a docência em enfermagem, ainda nesta área, visou identificar preditores da intenção de professores permanecerem na instituição de ensino e identificou o suporte da administração e a expertise percebida no ensino como variáveis dessa predição(19).

Em relação à administração dos serviços de enfermagem, os demais estudos que utilizaram a MEE exploraram efeitos da organização do trabalho sobre: o comprometimento, satisfação, empoderamento, efetividade e confiança profissional ${ }^{(9,11,22)}$; as características que contribuem para adesão ao uso de tecnologias de informação ${ }^{(13,15,21,24,28)}$; a identidade e relacionamento interpessoal ${ }^{(16)}$. Ainda com este foco, estudos envolvendo saúde do trabalhador contemplaram a análise de preditores de Burnout ${ }^{(14,17)}$, 
estresse $^{(23,25)}$ e dores musculoesqueléticas ${ }^{(26)}$. Os achados apontam que a justiça organizacional aumenta a confiança e a identificação do profissional com a organização, elementos que fortalecem o compromisso com a mesma ${ }^{(9)}$; e que as características do trabalho, a liderança transformacional e o empoderamento aumentam efetividade laboral ${ }^{(11)}$, sendo esse um fator determinante para a satisfação no trabalho ${ }^{(22)}$. Na área de tecnologia, observa-se que enfermeiras otimistas, inovadoras, seguras em relação a tecnologia apresentam maior facilidade para uso dos sistemas móveis de informação em enfermagem e percebem-nos como úteis ao processo de trabalho, o que contribui para a adesão a estes sistemas e para o bom desempenho da equipe de enfermagem ${ }^{(13,24)}$. A intenção em aderir aos sistemas e a consequente melhora dos resultados do trabalho de enfermagem também sofre a ação de fatores como, a influência social; desempenho esperado e a compatibilidade tecnológica com o processo de trabalho; as condições de uso; as funcionalidades de suporte oferecidas; e o esforço necessário para conhecer e utilizar o sistema ${ }^{(15,21,28)}$. Os estudos voltados à saúde do trabalhador demonstram que o enfermeiro com pouca autonomia e controle sobre o ambiente, pouca confiança no líder e com piores relações com o médico apresenta maior nível de exaustão emocional ${ }^{(14,17)}$ e que a intenção de deixar o trabalho e a confiança no líder contribuíram para o aumento do cinismo ${ }^{(14,17)}$ e redução da eficácia profissional ${ }^{(17)}$. Por outro lado, os indivíduos Hardy apresentaram baixos níveis de estresse, são mais acessíveis, têm um estilo de controle interno e confiança na resolução de problemas $^{(23)}$. Verificou-se também que a depressão, distúrbios na coluna, idade, sindicalização, demandas físicas no local de trabalho e baixo controle no trabalho aumentam a severidade e interferência da dor durante o trabalho, o que por sua vez impacta na duração da ausência ao trabalho(26).

\section{DISCUSSÃO}

A pesquisa é construída e desenvolvida com o intuito de servir como alicerce do conhecimento aos pesquisadores e a sociedade em geral, ser utilizada para leitura crítica e motivar reflexões sobre a prática da profissão ${ }^{(29)}$. Nesse sentido, a produção de investigações que usam a MEE auxilia na compreensão das diversas relações causais, explicando a forma como os fenômenos/variáveis se relacionam para produzir um dado desfecho ${ }^{(9-28,30)}$.

Observou-se que, embora a enfermagem brasileira apresente número considerável de publicações nacionais e internacionais, a prevalência de pesquisas publicadas que usaram a MEE, como tipo específico de análise ocorreu em países da Ásia, América do Norte e Europa. Portanto, observa-se a necessidade de manter uma postura de abertura as novas possibilidades de investigação e análise, tendo em vista a atuação com competência e segurança $a^{(31)}$.

O uso da MEE, devido a suas características metodológicas permite aprimorar alguns instrumentos de medida utilizados pela enfermagem e auxilia a explicação de alguns fenômenos ${ }^{(2)}$ que foram investigados em outros países, mas que também inquietam pesquisadores brasileiros. Neste sentido a MEE permite ratificar a estrutura fatorial das escalas de medida utilizadas nas diferentes amostras de sujeitos brasileiros bem como testar as relações entre variáveis latentes e observadas ${ }^{(1-2)}$. 
Analisando-se os dados das pesquisas desta Revisão Integrativa, verifica-se a preocupação com o impacto do processo de trabalho na saúde do trabalhador de enfermagem.

Os pesquisadores apontam que as condições de trabalho desfavoráveis causam o adoecimento do trabalhador, incluindo a ocorrência de estresse e Burnout, o que reduz a produtividade, eleva os custos com absenteísmo, rotatividade e afastamentos do pessoal e afeta a qualidade do cuidado ${ }^{(14,16-17,31)}$. Mas, também apontam que a identificação com a equipe atenua o sentimento de impotência e que a disposição para manejar conflitos e a impotência mediam a capacidade de interação e estabilidade laboral ${ }^{(16)}$. Igualmente apontam que o cinismo, subescala do Inventário de Burnout, media a confiança no líder e a liderança percebida do funcionário ${ }^{(17)}$. Deste modo demonstra os efeitos das variáveis, ou de seus componentes, entre si e como se comportam no modelo hipotético testado.

Sendo assim, tanto pesquisadores como enfermeiros da prática assistencial dispõem de elementos específicos para propor medidas que tornem relação do profissional de enfermagem com o trabalho mais saudável e menos desgastante, o que impactará na saúde do trabalhador e em consequência nos resultados institucionais.

Ainda na área organizacional, parte dos estudos analisou os fatores que afetam a adesão dos profissionais aos sistemas de informação em enfermagem, como prontuários eletrônicos e sistemas móveis de informação e registros ${ }^{(13,15,21,24,28)}$. A implantação da tecnologia de informação em saúde tem sido preconizada e estimulada nos últimos anos como instrumento para incrementar a segurança do paciente, a qualidade dos serviços de saúde e reduzir custos ${ }^{(13,15)}$. No entanto, apesar dos altos custos de implantação desses sistemas, os serviços têm enfrentado dificuldades quanto a sua adesão e uso por parte dos profissionais. Nesse sentido, os pesquisadores identificaram diferentes fatores comportamentais e relativos ao próprio sistema que contribuem para a compreensão e adesão aos sistemas informatizados em saúde ${ }^{(13,15,21,24,28)}$. A partir disso, modificações nos processos desenvolvimento e implantação, incluindo revisão das funcionalidades, compatibilidade, serviços de suporte, facilidade de uso, poderão melhorar a adesão dos usuários, aumentando a qualidade de assistência de enfermagem.

No que tange ao ensino e assistência de enfermagem, os estudos com a MEE confirmaram que a liderança e o compromisso com a enfermagem causam satisfação aos estudantes, que a autorreflexão e o insight os protegem do estresse e que a autoeficácia prediz o desempenho profissional das enfermeiras. Ainda que o suporte da administração além de favorecer a permanência dos professores nas instituições de ensino, quando coligado ao feedback, melhora a prática de lavagem das mãos. Observa-se, portanto, que as investigações ora analisadas apresentam elementos que podem subsidiar as boas praticas da enfermagem.

\section{CONCLUSÃO}

Nessa revisão, verificou-se o aumento do número de estudos que aplicaram a MEE na área de enfermagem nos últimos anos, especialmente no processo de organização do trabalho, com nível de Evidência 5 e desenvolvidos em países da Ásia, América do Norte e Europa. 
O notável enfoque dos estudos sobre a compreensão dos fatores do ambiente de trabalho que afetam o comprometimento, a satisfação e a confiança na organização, o empoderamento, a efetividade e o relacionamento interpessoal evidenciam a importância das condições laborais para a saúde do profissional de enfermagem e para o funcionamento da organização e manutenção dos profissionais na instituição. Isso porque condições de trabalho desfavoráveis causam o adoecimento do trabalhador, com elevação de custos decorrentes de absentismo e afastamentos, bem como redução da qualidade do cuidado prestado pela enfermagem. Nesse sentido, a técnica tem contribuído para a explicação de um grupo de relações que auxiliam a compreender alguns desfechos presentes no cotidiano laboral de enfermagem. Com estes elementos será possível desenvolver medidas preventivas mais pontuais e possivelmente, obter maior efetividade.

Essas contribuições também são válidas para os modelos analisados nos campos de ensino e assistência de enfermagem, porém a aplicação da modelagem nesses processos ainda é escassa. Nesse sentido, incentiva-se a construção de modelos hipotéticos voltados a essas áreas, visto que apresentam alguns objetos que apesar de bastante investigados, ainda carecem de soluções, como é o caso da adesão à lavagem das mãos. Por isso, é importante que os pesquisadores brasileiros se apropriem dessa técnica de análise e que desenvolvam estudos com delineamentos de maior poder explicativo.

A MEE é uma técnica que pode propiciar a ampliação do campo teórico e metodológico da enfermagem, mas como qualquer outra, não é capaz de abranger todos os tipos de objetos de pesquisa. Uma das limitações a seu uso é o tipo de análise estatística utilizada atualmente a qual prevê avaliar exclusivamente relações lineares entre variáveis. Outro limite a seu uso é o tamanho das amostras. Isso porque a MEE exige variabilidade acentuada dos dados para realizar o processo de estimativa. Mesmo assim esta técnica consegue auxiliar pesquisadores a explicarem alguns fenômenos que causam inquietação na enfermagem.

\section{REFERÊNCIAS}

1. Hair JF, Black WC, Babin BJ, Anderson RE, Tatham RL. Análise Multivariada de Dados. 6a ed. Porto Alegre: Bookman; 2009.

2. Pilati R, Laros JA. Modelos de equações estruturais em psicologia: conceitos e aplicações. Psicol Teor e Pesqui [Internet]. 2007 [acesso em: 31 dez. 2017];23(2):205-16. Disponível em: http://doi.org/10.1590/S010237722007000200011.

3. Unruh L, Zhang NJ, Chisolm L. Job and Professional Leaving Among Newly Licensed RNs: A Structural Equation Model. West J Nurs Res [Internet]. 2016 [acesso em: 31 dez. 2017];38(1):5-26. Disponível em: http://doi.org/10.1177/0193945914559290.

4. Maillet É, Mathieu L, Sicotte C. Modeling factors explaining the acceptance, actual use and satisfaction of nurses using an Electronic Patient Record in acute care settings: An extension of the UTAUT. Int J Med Inform [Internet]. 2015 [acesso em: 31 dez. 2017];84(1):36-47. Disponível em: http://doi.org/10.1016/j.ijmedinf.2014.09.004.

5. Mendes KDS, Silveira RCCP, Galvão CM. Revisão integrativa: método de pesquisa para a incorporação de evidências na saúde e na enfermagem. Texto Context - Enferm [Internet]. 2008 [acesso em: 31 dez. 2017];17(4):758-64.

Disponível em: http://doi.org/10.1590/S0104-07072008000400018. 
6. Ursi ES, Gavão CM. Prevenção de lesões de pele no perioperatório: revisão integrativa da literatura. Rev Lat Am Enfermagem [Internet]. 2006 [acesso em: 31 dez. 2017];14(1):124-31. Disponível em: http://doi.org/10.1590/s010411692006000100017.

7. Malta M, Cardoso LO, Bastos FI, Magnanini MMF, Silva CMFP. Iniciativa STROBE: subsídios para a comunicação de estudos observacionais. Rev Saude Publica [Internet]. 2010 [acesso em: 31 dez. 2017];44(3):559-65. Disponível em: http://doi.org/10.1590/S0034-89102010000300021.

8. Stetler CB, Morsi D, Rucki S, Broughton S, Corrigan B, Fitzgerald J, et al. Utilization-focused integrative reviews in a nursing service. Appl Nurs Res [Internet]. 1998 [acesso em: 31 dez. 2017];11(4):195-206. Disponível em:

http://doi.org/10.1016/S0897-1897(98)80329-7.

9. Chen S-Y, Wu W-C, Chang C-S, Lin C-T, Kung J-Y, Weng H-C, et al. Organizational justice, trust, and identification and their effects on organizational commitment in hospital nursing staff. BMC Health Serv Res [Internet]. 2015 [acesso em: 31 dez. 2017];15(1):363. Disponível em: http://doi.org/10.1186/s12913-015-1016-8.

10. Herrera-Usagre M, Pérez-Pérez P, Vázquez-Vázquez M, Santana-López V. Profesionales de salud ante la mejora de la higiene de las manos: estrategias clásicas versus estrategias avanzadas. Rev Chil infectología [Internet]. 2014 [acesso em: 31 dez. 2017];31(5):534-41. Disponível em: http://doi.org/10.4067/S0716-10182014000500004.

11. Eo Y-S, Kim Y-H, Lee N-Y. Path Analysis of Empowerment and Work Effectiveness among Staff Nurses. Asian Nurs Res (Korean Soc Nurs Sci) [Internet]. 2014 [acesso em: 31 dez. 2017];8(1):42-8. Disponível em:

http://doi.org/10.1016/j.anr.2014.02.001.

12. Zhu DQ, Norman IJ, While AE. Nurses' self-efficacy and practices relating to weight management of adult patients: a path analysis. Int J Behav Nutr Phys Act [Internet]. 2013 [acesso em: 31 dez. 2017];10:131. Disponível em: http://doi.org/10.1186/1479-5868-10-131.

13. Kuo K-M, Liu C-F, Ma C-C. An investigation of the effect of nurses' technology readiness on the acceptance of mobile electronic medical record systems. BMC Med Inform Decis Mak [Internet]. 2013 [acesso em: 31 dez.

2017];13(1):88. Disponível em: http://doi.org/10.1186/1472-6947-13-88.

14. Panunto MR, Guirardello E de B. Professional nursing practice: environment and emotional exhaustion among intensive care nurses. Rev Lat Am Enfermagem [Internet]. 2013 [acesso em: 31 dez. 2017];21(3):765-72. Disponível em: http://doi.org/10.1590/S0104-11692013000300016.

15. Lee C-C, Lin S-P, Yang S-L, Tsou M-Y, Chang K-Y. Evaluating the influence of perceived organizational learning capability on user acceptance of information technology among operating room nurse staff. Acta Anaesthesiol Taiwanica [Internet]. 2013 [acesso em: $31 \mathrm{dez.} \mathrm{2017];51(1):22-7.} \mathrm{Disponível} \mathrm{em:}$

http://doi.org/10.1016/j.aat.2013.03.013.

16. Moreland JJ, Ewoldsen DR, Albert NM, Kosicki GM, Clayton MF. Predicting Nurses' Turnover: The Aversive Effects of Decreased Identity, Poor Interpersonal Communication, and Learned Helplessness. J Health Commun [Internet]. 2015 [acesso em: 31 dez. 2017];20(10):1155-65. Disponível em: http://doi.org/10.1080/10810730.2015.1018589. 17. Bobbio A, Manganelli AM. Antecedents of hospital nurses' intention to leave the organization: A cross sectional survey. Int J Nurs Stud [Internet]. 2015 [acesso em: 31 dez. 2017];52(7):1180-92. Disponível em::

http://doi.org/10.1016/j.ijnurstu.2015.03.009.

18. D'Souza MS, Karkada SN, Parahoo K, Venkatesaperumal R. Perception of and satisfaction with the clinical learning environment among nursing students. Nurse Educ Today [Internet]. 2015 [acesso em: 31 dez. 2017];35(6):833-40. Disponível em: http://doi.org/10.1016/j.nedt.2015.02.005.

19. Candela L, Gutierrez AP, Keating S. What predicts nurse faculty members' intent to stay in the academic organization? A structural equation model of a national survey of nursing faculty. Nurse Educ Today [Internet]. 2015 [acesso em: 31 dez. 2017];35(4):580-9. Disponível em: http://doi.org/10.1016/j.nedt.2014.12.018.

20. Eng C-J, Pai H-C. Determinants of nursing competence of nursing students in Taiwan: The role of self-reflection and insight. Nurse Educ Today [Internet]. 2015 [acesso em: 31 dez. 2017];35(3):450-5. Disponível em:

http://doi.org/10.1016/j.nedt.2014.11.021.

21. Maillet É, Mathieu L, Sicotte C. Modeling factors explaining the acceptance, actual use and satisfaction of nurses using an Electronic Patient Record in acute care settings: An extension of the UTAUT. Int J Med Inform [Internet]. 2015 [acesso em: 31 dez. 2017];84(1):36-47. Disponível em: http://doi.org/10.1016/j.ijmedinf.2014.09.004.

22. Spence Laschinger HK, Nosko A, Wilk P, Finegan J. Effects of unit empowerment and perceived support for professional nursing practice on unit effectiveness and individual nurse well-being: A time-lagged study. Int J Nurs

Rev. Eletr. Enf. [Internet]. 2017 [acesso em:_____];19:a51. Disponível em: http://doi.org/10.5216/ree.v19.42988. 
Stud [Internet]. 2014 [acesso em: 31 dez. 2017];51(12):1615-23. Disponível em:

http://doi.org/10.1016/j.ijnurstu.2014.04.010.

23. Abdollahi A, Talib MA, Yaacob SN, Ismail Z. Problem-Solving Skills and Hardiness as Protective Factors against Stress in Iranian Nurses. Issues Ment Health Nurs [Internet]. 2014 [acesso em: 31 dez. 2017];35(2):100-7. Disponível em: http://doi.org/10.3109/01612840.2013.843621.

24. Chiu Y-L, Tsai C-C. The roles of social factor and internet self-efficacy in nurses' web-based continuing learning. Nurse Educ Today [Internet]. 2014 [acesso em: 31 dez. 2017];34(3):446-50. Disponível em:

http://doi.org/10.1016/j.nedt.2013.04.013.

25. Dotson MJ, Dave DS, Cazier JA, Spaulding TJ. An Empirical Analysis of Nurse Retention. JONA J Nurs Adm [Internet]. 2014 [acesso em: 31 dez. 2017];44(2):111-6. Disponível em: http://doi.org/10.1097/NNA.0000000000000034.

26. Murray E, Franche R-L, Ibrahim S, Smith P, Carnide N, Côté P, et al. Pain-Related Work Interference is a Key Factor in a Worker/Workplace Model of Work Absence Duration Due to Musculoskeletal Conditions in Canadian Nurses. J Occup Rehabil [Internet]. 2013 [acesso em: 31 dez. 2017];23(4):585-96. Disponível em:

http://doi.org/10.1007/s10926-012-9408-7.

27. Valdez LP, de Guzman A, Escolar-Chua R. A structural equation modeling of the factors affecting student nurses' medication errors. Nurse Educ Today [Internet]. 2013 [acesso em: 31 dez. 2017];33(3):222-8. Disponível em:

http://doi.org/10.1016/j.nedt.2012.01.001.

28. Hsiao J-L, Chen R-F. An Investigation on Task-Technology Fit of Mobile Nursing Information Systems for Nursing Performance. CIN Comput Informatics, Nurs [Internet]. 2012 [acesso em: 31 dez. 2017];30(5):265-73. Disponível em: http://doi.org/10.1097/NCN.0b013e31823eb82c.

29. Silva RM, Peres RR, Camponogara S, Brum CN. A produção científica brasileira sobre cuidados de enfermagem a pacientes portadores de úlceras por pressão. Revista de Enfermagem da UFSM [Internet]. 2011 [acesso em: 31 dez. 2017];1(2):246-53. Disponível em: https://periodicos.ufsm.br/reufsm/article/view/2486.

30. Hulley SB, Cumming SR, Browner WS, Grady DG, Hearst NB, Newman TB. Delineando a pesquisa clínica: uma abordagem epidemiológica. Porto Alegre: Artmed; 2008. 384 p.

31. Pires DEP. Transformações necessárias para o avanço da Enfermagem como ciência do cuidar. Rev Bras Enferm [Internet]. 2013 [acesso em: 31 dez. 2017];66(esp.):39-44. Disponível em: http://doi.org/10.1590/S003471672013000700005. 